\title{
Predicting Extrathyroidal Extension in Patients With Papillary Thyroid Microcarcinoma According to a BRAF Mutation
}

\author{
Doh Young Lee ${ }^{1,2} \cdot$ Soo Min Hwang ${ }^{1} \cdot$ Jee Hyun An²,3 $\cdot$ Kyu Ri Son ${ }^{2,4} \cdot$ Seung-Kuk Baek ${ }^{1,2} \cdot$ Sin Gon Kim²,3 \\ Yang Seok Chae ${ }^{2,5} \cdot$ Kwang-Yoon Jung ${ }^{1,2}$ \\ ${ }^{1}$ Department of Otorhinolaryngology-Head and Neck Surgery, ${ }^{2}$ Thyroid Center, Departments of ${ }^{3}$ Internal Medicine, ${ }^{4}$ Radiology, and ${ }^{5}$ Pathology, \\ Korea University Anam Hospital, Korea University College of Medicine, Seoul, Korea
}

Objectives. The aim of this study was to evaluate the association between preoperative parameters and extrathyroidal extension (ETE) of papillary thyroid microcarcinoma (PTMC) according to the BRAF mutation and to evaluate the preoperative predictability of ETE.

Methods. We analyzed the medical records of 332 patients with PTMC (140 in the BRAF- group and 192 in the BRAF+ group). The presence of ETE was subjected to a correlation analysis with age, sex, tumor size, clinical nodal status, and ultrasonography (US) findings. Among the US findings, the correlation between tumors and the thyroid capsule was categorized into four groups; US group A, intraparechymal; US group B, tumor abutting the capsule $<50 \%$ of diameter; US group C, tumor abutting $>50 \%$ of diameter; and US group D, tumor destroyed the capsule. The predictive value of ETE, including sensitivity, specificity, and positive and negative predictive values were evaluated.

Results. Tumor size and US group were significantly correlated with gross ETE in the BRAF- and BRAF+ groups. Tumor size of $0.5 \mathrm{~cm}$ and US groups $\mathrm{B}$ and $\mathrm{C}$ in the BRAF- group were cutoff values for gross ETE, with a negative predictive value of $100 \%$, whereas tumor size of $0.7 \mathrm{~cm}$ and US groups A and B in the BRAF+ group had negative predictive values of $92.4 \%$ and $100 \%$, respectively.

Conclusion. Excluding of ETE by US was categorized according to tumor size and US findings. A different categorization to exclude ETE is needed according to the BRAF mutation.

Keywords. Papillary Thyroid Microcarcinoma; BRAF Mutation; Extrathyroidal Extension; Capsules; Size

\section{INTRODUCTION}

The BRAF mutation is associated with a worse initial presentation and prognosis of papillary thyroid carcinoma (PTC) [1,2]. Among several clinicopathological features, extrathyroidal extension (ETE) is significantly associated with the BRAF muta-

- Received October 27, 2015

Revised March 16, 2016

Accepted March 24, 2016

- Corresponding author: Kwang-Yoon Jung

Department of Otolaryngology-Head and Neck Surgery, Korea University

College of Medicine, 73 Inchon-ro, Seongbuk-gu, Seoul 02841, Korea

Tel: +82-2-920-5486, Fax: +82-2-925-5233

E-mail: kyjungmd@gmail.com tion not only in PTC but in papillary thyroid microcarcinoma (PTMC) [3-6]. Considering that ETE is a key risk stratification and treatment planning parameter, predicting ETE preoperatively is important to determine surgical extent, particularly in patients with PTMC [7-9]. No mixed analysis of the BRAF mutation and predicting ETE had not been reported until recently, although several studies have focused on the association between preoperative ultrasonography (US) findings and postoperative ETE in pathological findings [10,11].

Given that ETE can differ according to the presence of the BRAF mutation, a different strategy to predict ETE is necessary. In addition, because microscopic ETE has minimal clinical implications and significance, predicting gross ETE can be of more

Copyright (C) 2017 by Korean Society of Otorhinolaryngology-Head and Neck Surgery.

This is an open-access article distributed under the terms of the Creative Commons Attribution Non-Commercial License (http://creativecommons.org/licenses/by-nc/4.0)

which permits unrestricted non-commercial use, distribution, and reproduction in any medium, provided the original work is properly cited. 

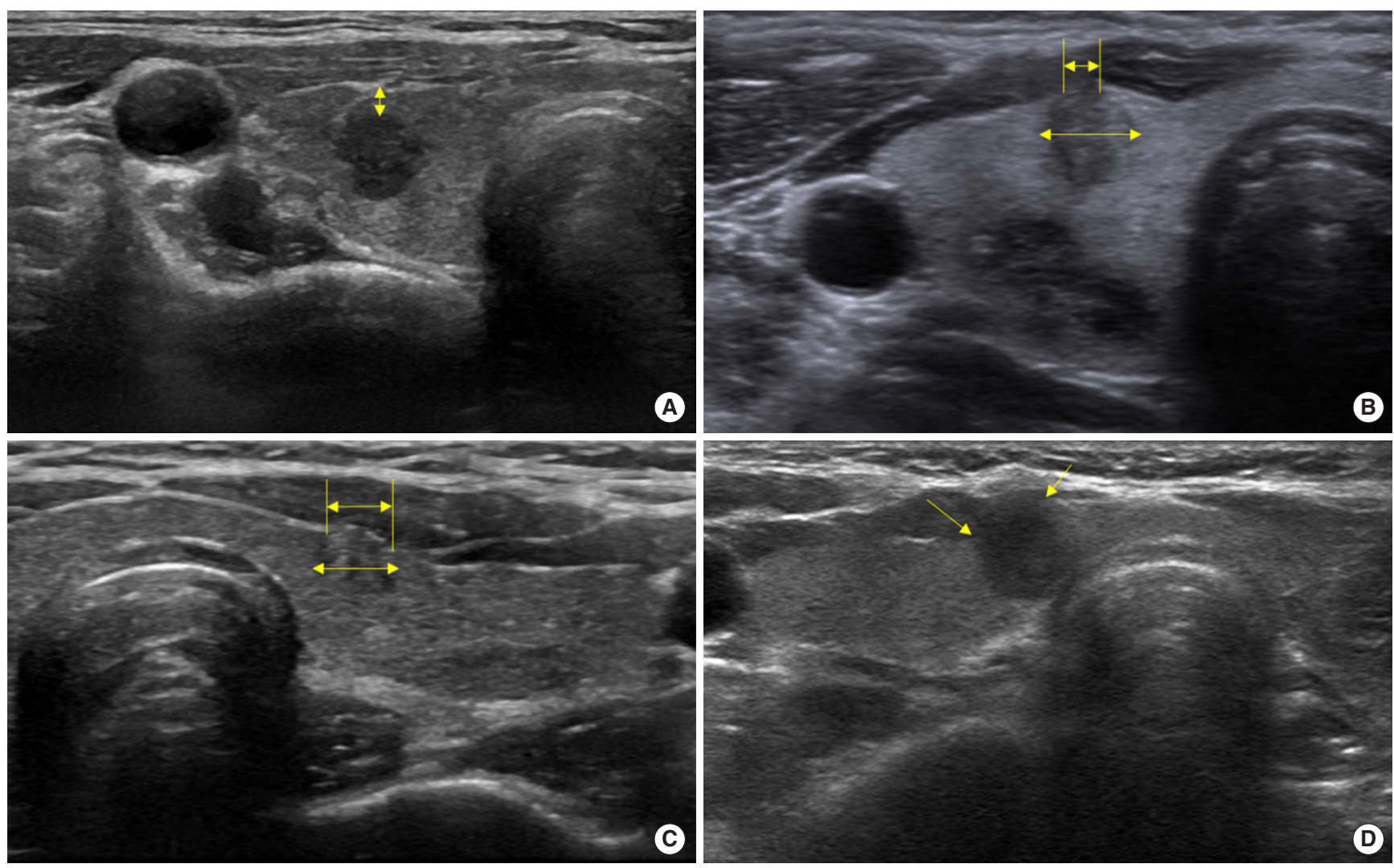

Fig. 1. Categorizing tumors according to their relationship with the thyroid capsule on preoperative ultrasonography. (A) Intraparechymal (arrow, thyroid parenchym between capsule and tumor), (B) abutting $<50 \%$ of tumor diameter (lower arrow, tumor size; upper arrow, abutting length), (C) abutting $>50 \%$ of tumor diameter (lower arrow, tumor size; upper arrow, abutting length), and (D) tumor destroying the capsule (arrows).

benefit. Therefore, in this study, we assessed preoperative parameters associated with gross ETE separately in BRAF positive and negative tumors and evaluated the predictability of gross ETE using important preoperative parameters.

\section{MATERIALS AND METHODS}

\section{Patients}

All protocols and the experimental design were approved by the Institutional Review Board of the Korea University College of

\section{H I}

- Extrathyroidal extension (ETE) of papillary thyroid microcarcinoma (PTMC) is predictable by the combinations of BRAF mutation, tumor size and ultrasonography findings.

- ETE is excluded in BRAF-negative PTMC with size $<0.7 \mathrm{~cm}$ and capsular abutting $<50 \%$.

- ETE is excluded in BRAF-positive PTMC with size $<0.5 \mathrm{~cm}$ and no capsular abutting.

- Preoperative prediction of ETE might contribute to determine surgical extent for PTMC.
Medicine (IRB no. ED15212). The medical records of patients undergoing total thyroidectomy (with or without neck dissection) in the Department of Otolaryngology-Head and Neck Surgery, Korea University Medical Center between September 2011 and June 2014 were reviewed retrospectively. Patients with diagnoses other than PTMC were excluded, as were those with tumors $>1 \mathrm{~cm}$, mixed tumors, and those with missing US data. The parameters investigated were patient age, sex, BRAF mutation analysis of aspirated cytology sample, preoperative US findings, and pathological reports. BRAF mutation analysis was performed by polymerase chain reaction and pyrosequencing as described elsewhere [12]. Thyroid nodules were evaluated using standard US methodology, including size, composition, echogenicity of the solid tissue, orientation, shape, margin, and calcification. The pathologic reports were uniformly categorized into bilaterality, multicentricity, surgical margin, lymphovascular invasion, gross and/or microscopic ETE, and the presence of nodal metastasis.

\section{Categorization according to tumor location and relation to the capsule}

We categorized the tumor characteristics into four US groups: US group A, tumor completely enveloped by thyroid parenchy- 
ma; US group B, tumor attached to the thyroid capsule without definite destruction of the capsule and contact area $<50 \%$ of tumor diameter; US group C, tumor attached to the thyroid capsule without definite destruction of the capsule and contact area $>50 \%$ of tumor diameter; and US group D, tumor attached to the thyroid capsule with loss of capsule shadow (Fig. 1).

\section{Statistical analysis}

Continuous outcomes were analyzed using independent $t$-tests between two groups, and dichotomous outcomes were analyzed using the chi-square test for trends. A binary logistic regression analysis was performed to assess the correlations between the preoperative parameters and ETE. A receiver-operating characteristic (ROC) curve analysis was performed to evaluate accuracy in predicting ETE. Indicators of predictive performance, including sensitivity, specificity, positive predictive value, and negative predictive value were calculated for the categories of preoperative parameters. All statistical analyses were performed using IBM SPSS ver. 20.0 (IBM Co.,Armonk, NY, USA). A P-value less than 0.05 was considered significant.

\section{RESULTS}

\section{Difference according to the BRAF mutation}

A total of 332 patients were enrolled in this study. Mean age was $52.5 \pm 11.0$ years, and the male to female ratio was 1:4.53. The pre- and postoperative parameters were compared between the two groups according to BRAF mutation status (140 patients in BRAF- group and 192 patients in BRAF+ group) (Table 1). Males were predominant and tumors were larger in the BRAF+ group than those in the BRAF- group $(P=0.001$ and $P<0.001$, respectively). Suspicious lymph node metastasis was also prevalent in the BRAF+ group, but a significant difference was observed only for central node metastasis $(P=0.032)$. US group A was more prevalent in the BRAF- group, whereas US groups $\mathrm{B}$ $\mathrm{D}$ were more prevalent in $\mathrm{BRAF}+$ group $(P=0.028)$. The "taller than wide" US feature was more common in the BRAF+ group than that in the BRAF- group, and central node metastasis, positive resection margin $(P<0.001)$, and bilaterality were more common in the BRAF+ group in the pathological report $(P<$ $0.001, P=0.014$, and $P=0.031$, respectively). Both microscopic and gross ETE were more prevalent in the BRAF+ group than

Table 1. Perioperative data according to the BRAF mutation

\begin{tabular}{|c|c|c|c|}
\hline Variable & $\operatorname{BRAF}(-)(n=140)$ & $\operatorname{BRAF}(+)(n=192)$ & $P$-value \\
\hline Age (yr) & $53.6 \pm 11.0$ & $51.8 \pm 11.0$ & 0.155 \\
\hline Sex (male:female) & $1: 5.1$ & $1: 4.1$ & $0.001^{*}$ \\
\hline \multicolumn{4}{|l|}{ Preoperative finding } \\
\hline Size $(\mathrm{cm})$ & $0.53 \pm 0.18$ & $0.65 \pm 0.19$ & $<0.001^{*}$ \\
\hline cN1a & $126(90.0)$ & $182(95.8)$ & $0.032^{*}$ \\
\hline $\mathrm{cN} 1 \mathrm{~b}$ & $4(2.9)$ & $12(6.3)$ & 0.116 \\
\hline Grouping according to the correlation of tumor and capsule & & & $0.028^{*}$ \\
\hline A & $58(41.4)$ & $56(29.2)$ & \\
\hline B & $22(15.7)$ & $54(28.1)$ & \\
\hline C & $38(27.1)$ & $50(26.0)$ & \\
\hline $\mathrm{D}$ & $22(15.7)$ & $32(16.7)$ & \\
\hline \multicolumn{4}{|l|}{ Preoperative ultrasonographic feature } \\
\hline Marked hypoechoic & $114(81.4)$ & $156(81.3)$ & 0.542 \\
\hline Taller than wide & $80(57.1)$ & $144(75.0)$ & $<0.001^{*}$ \\
\hline Spiculated margin & $94(67.1)$ & $142(74.0)$ & 0.110 \\
\hline Microcalcification & $68(48.6)$ & $102(53.1)$ & 0.239 \\
\hline \multicolumn{4}{|l|}{ Pathologic finding } \\
\hline Central node metastasis & $42(30.0)$ & $94(49.0)$ & $<0.001^{*}$ \\
\hline Lateral node metastasis & $2(1.4)$ & $10(5.2)$ & 0.059 \\
\hline Positive surgical margin & $4(2.9)$ & $18(9.4)$ & $0.014^{*}$ \\
\hline Bilaterality & $30(21.4)$ & $60(31.3)$ & $0.031^{*}$ \\
\hline Multifocality & $54(38.6)$ & $82(42.7)$ & 0.260 \\
\hline Lymphatic invasion & 0 & $4(2.1)$ & 0.110 \\
\hline Vascular invasion & 0 & $2(1.0)$ & 0.334 \\
\hline Extrathyroidal extension & & & $0.001^{*}$ \\
\hline Microscopic & $38(27.1)$ & $76(39.6)$ & \\
\hline Gross & $8(5.7)$ & $26(13.5)$ & \\
\hline
\end{tabular}

Values are presented as mean \pm standard deviation or number (\%).

${ }^{\star} P<0.05$. 
those in the BRAF- group $(P=0.001)$.

\section{Correlations between extrathyroidal extension and the preop- erative parameters}

Tumor size and US group were significantly correlated with overall ETE in the BRAF- group $(P=0.012$ and $P=0.012$, respectively), whereas sex, tumor size, clinically suspicious lateral lymph node metastasis, and US group were significantly correlated in the BRAF+ group $(P=0.001,0.002,0.044$, and 0.020 , respectively) (Supplementary Table 1). Tumor size and US group were significantly correlated with gross ETE in the BRAF- and $\mathrm{BRAF}+$ groups (Table 2). The multivariate analysis revealed that both overall and gross ETE were significantly correlated with tumor size and US group in the BRAF- group. In the BRAF+ group, sex and tumor size were significantly correlated with overall ETE, whereas tumor size and US group were correlated with gross ETE (Table 3). Abutting site of the tumor and capsule on the US findings were significantly correlated with overall and gross ETE. However, a significant correlation was observed only in gross ETE in the BRAF- group after excluding tumors with an intraparechymal location (US group $\mathrm{A}, P=0.002$ ), whereas medial, lateral, or multiple contact showed a high prevalence of
ETE (Table 4).

\section{Predicting extrathyroidal extension according to the BRAF mutation}

Table 5 summarizes the ROC curve analysis that revealed the cutoff values for predicting ETE. Tumor size of $0.6 \mathrm{~cm}$ and US groups A and B were cutoff criteria for overall ETE in the BRAF- group, and tumor size of $0.7 \mathrm{~cm}$ and US groups B and C had a negative predictive value of $100 \%$ for gross ETE. Tumor size of $0.5 \mathrm{~cm}$ and US groups A and B were cutoff criteria for overall ETE in the BRAF+ group, whereas tumor size of $0.5 \mathrm{~cm}$ and US groups A and B had negative predictive value of $92.4 \%$ and $100 \%$ for gross ETE, respectively.

\section{DISCUSSION}

Our results demonstrate that the BRAF mutation is significantly correlated with ETE, and that size and US group are significant predictors of ETE in both the BRAF- and BRAF+ groups. The ETE prediction analysis included preoperative parameters and excluded postoperative findings because the preoperative pre-

Table 2. Univariate analysis for evaluating the correlation between gross extrathyroidal extension and preoperative parameters

\begin{tabular}{|c|c|c|c|c|}
\hline \multirow{2}{*}{ Parameters } & \multicolumn{2}{|c|}{$\operatorname{BRAF}(-)$} & \multicolumn{2}{|c|}{$\operatorname{BRAF}(+)$} \\
\hline & OR $(95 \% \mathrm{Cl})$ & $P$-value & OR $(95 \% \mathrm{Cl})$ & $P$-value \\
\hline Age & $0.982(0.921-1.048)$ & 0.586 & $0.991(0.955-1.028)$ & 0.629 \\
\hline Female sex & $3.333(0.605-18.371)$ & 0.167 & $0.945(0.355-2.516)$ & 0.910 \\
\hline Size & $62.294(47.328-819.917)$ & $<0.001^{*}$ & 39.837 (3.607-439.989) & $0.003^{*}$ \\
\hline cN1a & $1.432(0.143-4.856)$ & 0.690 & $1.115(0.131-9.449)$ & 0.921 \\
\hline $\mathrm{cN} 1 \mathrm{~b}$ & $0.621(0.314-6.648)$ & 0.539 & $2.246(0.566-8.912)$ & 0.250 \\
\hline US group & $2.692(1.217-5.956)$ & $0.015^{*}$ & $1.766(1.172-2.660)$ & $0.007^{*}$ \\
\hline Marked hypoechoic & $1.500(0.285-7.891)$ & 0.632 & $0.761(0.245-2.364)$ & 0.637 \\
\hline Taller than wide & $0.737(0.177-3.074)$ & 0.675 & $0.714(0.289-1.767)$ & 0.466 \\
\hline Spiculated margin & $0.467(0.111-1.957)$ & 0.297 & $2.108(0.689-6.450)$ & 0.191 \\
\hline Microcalcification & $1.062(0.255-4.428)$ & 0.934 & $1.488(0.638-3.471)$ & 0.357 \\
\hline
\end{tabular}

OR, odds ratio; $\mathrm{Cl}$, confidence interval; US, ultrasonography.

${ }^{*} P<0.05$.

Table 3. Multivariate analysis for extrathyroidal extension

\begin{tabular}{|c|c|c|c|c|}
\hline \multirow{2}{*}{ Parameter } & \multicolumn{2}{|c|}{$\operatorname{BRAF}(-)$} & \multicolumn{2}{|c|}{$\operatorname{BRAF}(+)$} \\
\hline & $\mathrm{OR}(95 \% \mathrm{Cl})$ & $P$-value & OR $(95 \% \mathrm{Cl})$ & $P$-value \\
\hline \multicolumn{5}{|c|}{ Overall extrathyroidal extension } \\
\hline Female sex & - & - & $0.282(0.132-0.604)$ & $0.001^{*}$ \\
\hline Size & $8.731(1.938-81.285)$ & $0.047^{*}$ & $10.076(1.604-63.308)$ & $0.014^{*}$ \\
\hline $\mathrm{cN} 1 \mathrm{~b}$ & - & - & $5.017(0.993-25.345)$ & 0.051 \\
\hline US group & $1.378(1.019-1.921)$ & $0.048^{*}$ & $1.137(0.832-1.554)$ & 0.421 \\
\hline \multicolumn{5}{|c|}{ Gross extrathyroidal extension } \\
\hline Size & $65.890(32.901-131.955)$ & $0.001^{*}$ & 17.860 (1.435-222.275) & $0.025^{*}$ \\
\hline US group & $2.730(1.092-7.517)$ & $0.025^{*}$ & $1.508(1.168-2.349)$ & $0.036^{*}$ \\
\hline
\end{tabular}

OR, odds ratio; $\mathrm{Cl}$, confidence interval; US, ultrasonography.

${ }^{*} P<0.05$. 
Table 4. Abbuting site and extrathyroidal extension

\begin{tabular}{|c|c|c|c|c|c|c|c|}
\hline \multirow{2}{*}{ Abutting site } & \multirow{2}{*}{ No. } & \multicolumn{3}{|c|}{ Extrathyroidal extension (total) } & \multicolumn{3}{|c|}{ Extrathyroidal extension (gross) } \\
\hline & & No. $(\%)$ & $P$-value ${ }^{\text {a) }}$ & $P$-value ${ }^{\text {b) }}$ & No. (\%) & $P$-value ${ }^{\mathrm{a})}$ & $P$-value ${ }^{\text {b) }}$ \\
\hline \multicolumn{8}{|l|}{$\operatorname{BRAF}(-)$} \\
\hline None & 58 & $10(17)$ & 0.024 & & 0 & $<0.001$ & \\
\hline Anterior & 52 & $24(46)$ & & 0.776 & $2(4)$ & & $0.002^{*}$ \\
\hline Posterior & 12 & $6(50)$ & & & 0 & & \\
\hline Medial & 4 & $2(50)$ & & & $2(50)$ & & \\
\hline Lateral & 8 & $2(25)$ & & & $2(25)$ & & \\
\hline Multiple & 6 & $2(33)$ & & & $2(33)$ & & \\
\hline \multicolumn{8}{|l|}{$\operatorname{BRAF}(+)$} \\
\hline None & 56 & $20(36)$ & 0.003 & & 0 & 0.001 & \\
\hline Anterior & 68 & $48(71)$ & & 0.080 & $14(21)$ & & 0.126 \\
\hline Posterior & 30 & $12(40)$ & & & $6(20)$ & & \\
\hline Medial & 10 & $6(60)$ & & & $2(20)$ & & \\
\hline Lateral & 18 & $10(56)$ & & & 0 & & \\
\hline Multiple & 10 & $6(60)$ & & & $4(40)$ & & \\
\hline
\end{tabular}

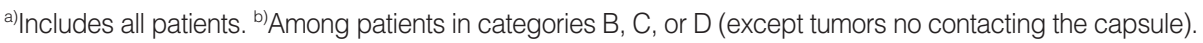

${ }^{*} P<0.05$.

Table 5. Predictability of extrathyroidal extension

\begin{tabular}{|c|c|c|c|c|c|c|c|}
\hline Variable & & Cutoff value $(\mathrm{cm})$ & Sensitivity (\%) & Specificity (\%) & PPV (\%) & NPV (\%) & $\operatorname{AUC}(95 \% \mathrm{Cl})$ \\
\hline \multicolumn{8}{|l|}{$\operatorname{BRAF}(-)$} \\
\hline \multirow[t]{2}{*}{ Overall extrathyroidal extension } & Size & 0.6 & 60.9 & 59.6 & 42.4 & 75.7 & $0.624(0.538-0.704)$ \\
\hline & US group & $A / B$ & 78.3 & 51.1 & 43.9 & 82.8 & $0.633(0.547-0.713)$ \\
\hline \multirow[t]{2}{*}{ Gross extrathyroidal extension } & Size & 0.7 & 100 & 56.1 & 12.1 & 100 & $0.845(0.774-0.900)$ \\
\hline & US group & $\mathrm{B} / \mathrm{C}$ & 100 & 60.6 & 13.3 & 100 & $0.777(0.698-0.843)$ \\
\hline \multicolumn{8}{|l|}{$\operatorname{BRAF}(+)$} \\
\hline \multirow[t]{2}{*}{ Overall extrathyroidal extension } & Size & 0.5 & 56.9 & 64.4 & 64.4 & 56.9 & $0.633(0.531-0.702)$ \\
\hline & US group & $A / B$ & 80.4 & 40.0 & 60.3 & 64.3 & $0.599(0.526-0.669)$ \\
\hline \multirow[t]{2}{*}{ Gross extrathyroidal extension } & Size & 0.5 & 61.5 & 73.5 & 26.7 & 92.4 & $0.703(0.633-0.767)$ \\
\hline & US group & $A / B$ & 100 & 33.7 & 19.1 & 100 & $0.666(0.595-0.733)$ \\
\hline
\end{tabular}

PPV, positive predictive value; NPV, negative predictive value; AUC, area under the curve; $\mathrm{Cl}$, confidence interval; US, ultrasonography.

diction is important to determine the treatment strategy including surgical extension. The predictive cutoff values for size and US group differed between the two groups. The size cutoff was larger for the BRAF- group, and the BRAF+ group had $100 \%$ negative predictive value for ETE in tumors with no capsular contact, whereas it was tumors with $<50 \%$ contact with the capsule in the BRAF- group.

In this study, the characteristics of BRAF+ tumors were similar to those reported previously. A meta-analysis showed that the BRAF mutation is associated with lymph node metastasis, stage, ETE, tumor size, male sex, multifocality, and absence of a capsule [3]. Our data also shows that the BRAF+ group had more male patients, larger tumors, clinical and pathological central node metastasis, bilaterality, and a positive resection margin in PTMC. One interesting finding was that tumors in the BRAF+ group tended to be located near the capsule, resulting in less proportion of US group A in the BRAF+ group. Moreover, the US showed that the BRAF+ group tended to have more malig- nant features than those in the BRAF- group, although statistical significance was observed only for the "taller than wide" characteristic. This result agrees with a previous finding that all malignant features are significantly more predominant in BRAFpositive tumors [13].

ETE is categorized as gross or microscopic depending on the extent of invasion. Outcomes from gross ETE are worse than those from microscopic ETE [14,15]. Recent studies have reported that microscopic ETE is not associated with increased recurrence or decreased survival in patients with PTC [16]. In addition, the insignificance of microscopic ETE is equally applied to PTMC, where it has no impact on recurrence in tumors $<1$ $\mathrm{cm}$ [17]. Although our study included an analysis of overall and gross ETE, only gross ETE may be clinically significant.

Several studies have evaluated the predictability of US findings for ETE $[10,18]$. Although these reports show relative good predictability of ETE, completely predicting positive or negative ETE has not been possible. Moreover, clinical application of US 
categories has also been difficult. Use of computed tomography, magnetic resonance imaging, and elastography have been suggested for better predictability and easy application in a clinical setting $[11,19,20]$, but complete prediction using preoperative findings remains difficult. In this study, we aimed to simplify US findings into four groups according to the correlation between the tumor and the thyroid capsule. Length of contact and the longest tumor diameter can be compared with a rough guess, resulting in faster tumor categorization using the US findings. Additionally, we considered BRAF mutation status to improve the predictive power with this simplified US grouping.

Several differences in ETE were detected between the BRAF+ and BRAF- groups. Tumor location was significantly correlated with gross ETE in the BRAF- group. Among tumors that contacted the capsule, medially or laterally located tumors had a higher incidence of gross ETE in the BRAF- group. No difference was found in the BRAF+ group according to tumor location, which necessitates further study. The reason why tumors medial or lateral abutting to the capsule had a higher incidence of ETE can be explained using a hypothesis from a previous report [11]. The US probe can compress the patient's neck and may slightly distort the normal parenchyma around the tumor, which makes the thyroid parenchyma thinner, particularly when the thyroid is pressed between a malignant tumor and the strap muscles. In contrast, the medial and lateral sides of the thyroid are relatively less compressed during a US examination, resulting in low false positive prediction of ETE.

ETE predictive power differed between the BRAF+ and BRAF- groups, as shown for the first time. The multivariate analysis revealed that tumor size and US group were significant predictors of ETE in both the BRAF- and BRAF+ groups. The best predictive ability was found for gross ETE in the BRAFgroup (area under the curve, 0.845 ). Considering that only gross ETE has a clinical impact, predicting gross ETE is more important than predicting overall ETE, as mentioned above. The negative predictive value in both groups was as high as $100 \%$ based on tumor size and US group. Therefore, we can exclude gross ETE in BRAF negative tumors $<0.7 \mathrm{~cm}$ with capsular contact $<50 \%$ of diameter. Similarly, gross ETE in BRAF positive tumors can also be excluded when the tumor is $<0.5 \mathrm{~cm}$ with no capsular contact. Consequently, ETE should be strictly excluded in the BRAF+ group with smaller sized tumors and an intraparenchymal location.

The BRAF mutation is associated with aggressiveness of PTC [21-23]. Thus, planning the surgical extent of PTC must be carefully considered. Because of the low mortality rate associated with PTC, particularly with PTMC, postoperative quality of life of patients who undergo thyroidectomies is as important as disease control [24]. Thus, several guidelines for treating differentiated thyroid cancer emphasize that surgical extent should decrease in patients in whom total thyroidectomy and lobectomy are expected to yield similar recurrence or survival rates. How- ever, negative prognosticators, such as ETE and lymph node metastasis, should be excluded when performing a lobectomy. Our results suggest that the BRAF mutation is key to determine the ETE exclusion criteria.

Although our study is valuable as the first study to evaluate the difference in predicting ETE according to the BRAF mutation, this study had several limitations. Firstly, this study has several repetitive demonstration of previous studies which show the BRAF mutation correlated with female gender, multifocality, ETE, lymph node metastasis, and TNM stage. Moreover, Kwak et al. [12] evaluated the association of BRAF mutation and its association with ultrasonographic features in Korean patients with PTMC. However, cutoff criteria was not shown which may be critical to preoperative decision on the extent of surgery. The most of the previous articles deals this matter, while no article show this categorization by BRAF mutation and ultrasonographic findings. Therefore, despite the several repetition in our study, our article can be valuable in that we categorize the preoperative feature and showed numerical cutoff value for helping decision making of the surgical extent. Second, a BRAF mutation preoperative analysis using fine needle aspiration cytology can be incorrect [25]. Thus, a false positive or negative BRAF mutation result could lead to an improper application. Third, this study involved microcarcinoma, which several guidelines suggest require no routine evaluation. Fourth, the degree of contact was assessed visually on preoperative US. Therefore, the results were subjective. Last, our study was based on a retrospective review, which has several inherent biases for analysis. A prospective study with a larger cohort may necessary to overcome this limitation.

In conclusion, our results suggest that the exclusion of ETE can be categorized according to the BRAF mutation, tumor size, and US findings. Tumors $<0.7 \mathrm{~cm}$ with contact $<50 \%$ of tumor diameter were factors affecting the absolute cutoff value in the BRAF- group for negatively predicting ETE. Tumors $<0.5 \mathrm{~cm}$ with no contact with the capsule were factors affecting the cutoff value in the BRAF+ group. Accordingly, a different categorization for excluding ETE is needed based on BRAF mutation status, and our data can be used a background data to decide surgical extent and to counsel patients.

\section{CONFLICT OF INTEREST}

No potential conflict of interest relevant to this article was reported.

\section{REFERENCES}

1. Frasca F, Nucera C, Pellegriti G, Gangemi P,Attard M, Stella M, et al. $\mathrm{BRAF}(\mathrm{V} 600 \mathrm{E})$ mutation and the biology of papillary thyroid cancer. 
Endocr Relat Cancer. 2008 Mar;15(1):191-205.

2. Guan H, Ji M, Bao R, Yu H, Wang Y, Hou P, et al. Association of high iodine intake with the T1799A BRAF mutation in papillary thyroid cancer. J Clin Endocrinol Metab. 2009 May;94(5):1612-7.

3. Li C, Lee KC, Schneider EB, Zeiger MA. BRAFV600E mutation and its association with clinicopathological features of papillary thyroid cancer: a meta-analysis. J Clin Endocrinol Metab. 2012 Dec;97(12): 4559-70.

4. Chakraborty A, Narkar A, Mukhopadhyaya R, Kane S, D’Cruz A, Rajan MG. BRAF V600E mutation in papillary thyroid carcinoma: significant association with node metastases and extra thyroidal invasion. Endocr Pathol. 2012 Jun;23(2):83-93.

5. Kim TH, Park YJ, Lim JA, Ahn HY, Lee EK, LeeYJ, et al. The association of the BRAF(V600E) mutation with prognostic factors and poor clinical outcome in papillary thyroid cancer: a meta-analysis. Cancer. 2012 Apr;118(7):1764-73.

6. Lee X, Gao M, Ji Y, Yu Y, Feng Y, Li Y, et al. Analysis of differential BRAF(V600E) mutational status in high aggressive papillary thyroid microcarcinoma. Ann Surg Oncol. 2009 Feb;16(2):240-5.

7. Byar DP, Green SB, Dor P,Williams ED, Colon J, van Gilse HA, et al. A prognostic index for thyroid carcinoma: a study of the E.O.R.T.C. Thyroid Cancer Cooperative Group. Eur J Cancer. 1979 Aug;15(8): 1033-41.

8. Hay ID, Grant CS, Taylor WF, McConahey WM. Ipsilateral lobectomy versus bilateral lobar resection in papillary thyroid carcinoma: a retrospective analysis of surgical outcome using a novel prognostic scoring system. Surgery. 1987 Dec;102(6):1088-95.

9. Hay ID, Bergstralh EJ, Goellner JR, Ebersold JR, Grant CS. Predicting outcome in papillary thyroid carcinoma: development of a reliable prognostic scoring system in a cohort of 1779 patients surgically treated at one institution during 1940 through 1989. Surgery. 1993 Dec;114(6):1050-7.

10. Kim SS, Lee BJ, Lee JC, Kim SJ, Lee SH, Jeon YK, et al. Preoperative ultrasonographic tumor characteristics as a predictive factor of tumor stage in papillary thyroid carcinoma. Head Neck. 2011 Dec; 33(12):1719-26.

11. Lee DY, Kwon TK, Sung MW, Kim KH, Hah JH. Prediction of extrathyroidal extension using ultrasonography and computed tomography. Int J Endocrinol. 2014;2014:351058.

12. Kwak JY, Kim EK, Chung WY, Moon HJ, Kim MJ, Choi JR. Association of BRAFV600E mutation with poor clinical prognostic factors and US features in Korean patients with papillary thyroid microcarcinoma. Radiology. 2009 Dec;253(3):854-60.

13. Kabaker AS, Tublin ME, Nikiforov YE, Armstrong MJ, Hodak SP, Stang MT, et al. Suspicious ultrasound characteristics predict BRAF
V600E-positive papillary thyroid carcinoma. Thyroid. 2012 Jun;22 (6):585-9.

14. Radowsky JS, Howard RS, Burch HB, Stojadinovic A. Impact of degree of extrathyroidal extension of disease on papillary thyroid cancer outcome. Thyroid. 2014 Feb;24(2):241-4.

15. Ito Y, Tomoda C, Uruno T, Takamura Y, Miya A, Kobayashi K, et al. Prognostic significance of extrathyroid extension of papillary thyroid carcinoma: massive but not minimal extension affects the relapse-free survival.World J Surg. 2006 May;30(5):780-6.

16. Jin BJ, Kim MK, Ji YB, Song CM, Park JH,Tae K. Characteristics and significance of minimal and maximal extrathyroidal extension in papillary thyroid carcinoma. Oral Oncol. 2015 Aug;51(8):759-63.

17. Moon HJ, Kim EK, Chung WY, Yoon JH, Kwak JY. Minimal extrathyroidal extension in patients with papillary thyroid microcarcinoma: is it a real prognostic factor? Ann Surg Oncol. 2011 Jul;18(7): 1916-23.

18. Kwak JY, Kim EK, Youk JH, Kim MJ, Son EJ, Choi SH, et al. Extrathyroid extension of well-differentiated papillary thyroid microcarcinoma on US.Thyroid. 2008 Jun;18(6):609-14.

19. Moon HJ, Kim EK, Yoon JH, Kwak JY. Clinical implication of elastography as a prognostic factor of papillary thyroid microcarcinoma. Ann Surg Oncol. 2012 Jul;19(7):2279-87.

20. King AD, Ahuja AT, To EW, Tse GM, Metreweli C. Staging papillary carcinoma of the thyroid: magnetic resonance imaging vs ultrasound of the neck. Clin Radiol. 2000 Mar;55(3):222-6.

21. Niederer-Wust SM, Jochum W, Forbs D, Brandle M, Bilz S, Clerici T, et al. Impact of clinical risk scores and BRAFV600E mutation status on outcome in papillary thyroid cancer. Surgery. 2015 Jan;157(1): 119-25.

22. Lim JY, Hong SW, Lee YS, Kim BW, Park CS, Chang HS, et al. Clinicopathologic implications of the BRAF(V600E) mutation in papillary thyroid cancer: a subgroup analysis of 3130 cases in a single center.Thyroid. 2013 Nov;23(11):1423-30.

23. Zheng X, Wei S, Han Y, LiY, YuY,Yun X, et al. Papillary microcarcinoma of the thyroid: clinical characteristics and BRAF(V600E) mutational status of 977 cases. Ann Surg Oncol. 2013 Jul;20(7):2266-73.

24. Ryu J, Ryu YM, Jung YS, Kim SJ, LeeYJ, Lee EK, et al. Extent of thyroidectomy affects vocal and throat functions: a prospective observational study of lobectomy versus total thyroidectomy. Surgery. 2013 Sep;154(3):611-20.

25. Leslie C, Grieu-Iacopetta F, Richter A, Platten M, Murray J, Frost FA, et al. BRAF p.Val600Glu (V600E) mutation detection in thyroid fine needle aspiration cell block samples: a feasibility study. Pathology. 2015 Aug;47(5):432-8. 
Lee DY et al. Prediction of Extrathyroidal Extension

Supplementary Table 1. Univariate analysis for evaluating the correlation between overall extrathyroidal extension and preoperative parameters

\begin{tabular}{|c|c|c|c|c|}
\hline \multirow{2}{*}{ Parameter } & \multicolumn{2}{|c|}{$\operatorname{BRAF}(-)$} & \multicolumn{2}{|c|}{$\operatorname{BRAF}(+)$} \\
\hline & $\mathrm{OR}(95 \% \mathrm{Cl})$ & $P$-value & OR $(95 \% \mathrm{Cl})$ & $P$-value \\
\hline Age & $0.983(0.952-1.015)$ & 0.298 & $0.985(0.960-0.011)$ & 0.264 \\
\hline Female sex & $0.800(0.237-2.702)$ & 0.719 & $0.288(0.142-0.587)$ & 0.001 \\
\hline Size & $15.143(1.798-127.547)$ & 0.012 & $13.188(2.615-66.502)$ & 0.002 \\
\hline cN1a & $0.868(0.274-2.756)$ & 0.811 & $1.116(0.271-4.600)$ & 0.879 \\
\hline $\mathrm{cN} 1 \mathrm{~b}$ & $0.674(0.068-6.664)$ & 0.736 & $4.889(1.041-22.950)$ & 0.044 \\
\hline US group & $1.505(0.095-2.070)$ & 0.012 & $1.384(1.052-1.822)$ & 0.020 \\
\hline Marked hypoechoic & $0.555(0.206-1.494)$ & 0.244 & $0.492(0.234-1.032)$ & 0.060 \\
\hline Taller than wide & $0.796(0.388-1.632)$ & 0.533 & $0.846(0.440-1.627)$ & 0.617 \\
\hline Spiculated margin & $1.515(0.724-3.173)$ & 0.270 & $0.941(0.493-1.794)$ & 0.853 \\
\hline Microcalcification & $1.240(0.612-2.512)$ & 0.551 & $1.164(0.660-2.056)$ & 0.599 \\
\hline
\end{tabular}

Overall extrathyroidal extension included microscopic and gross extrathyroidal extension.

OR, odds ratio; $\mathrm{Cl}$, confidence interval; US, ultrasonography. 